\title{
Escala de Evaluación del Comportamiento del Niño en su Versión para Padres (PRS):
}

\author{
Adaptación para su Uso en Buenos Aires \\ Molina, María Fernanda* ${ }^{1}$, Calero, Alejandra* \& Raimundi, María Julia* \\ * Consejo Nacional de Investigaciones Científicas y Tecnológicas. UBA-Instituto de Investigaciones de la \\ Facultad de Psicología de la Universidad de Buenos Aires. Buenos Aires, Argentina.
}

\begin{abstract}
Resumen. La evaluación psicológica infantil requiere de un proceso multifuente. Es por ello que contar con instrumentos que permitan captar la valoración que hacen los padres sobre las conductas y atributos del niño es de suma importancia. El objetivo de este trabajo es presentar los resultados de la adaptación y validación de la Escala de Evaluación del Comportamiento del Niño, en su versión para padres (PRS; Harter, 1985) para su uso en Buenos Aires (Argentina). Se trabajó con una muestra de 155 niños (edad $M=9.53 ; D E=1.40$ ) de ambos sexos de escuelas primarias de Buenos Aires y uno de sus padres. Se estudió la capacidad de discriminación de los ítems, la confiabilidad, validez de constructo, de contenido, convergente, y discriminante. Los resultados mostraron que este es un instrumento válido y confiable para evaluar la percepción que tienen los padres de niños de escuelas primarias de Buenos Aires de los atributos y competencias de sus hijos.
\end{abstract}

Palabras clave: Evaluación Psicológica Infantil; Escala; Comportamiento; Adaptación.

Parent's Rating Scale of Child's Actual Behavior (PRS): Adaptation for its Use in Buenos Aires

Abstract. The children psychological evaluation requires a multisource process. This is why having instruments that capture the valuation made by parents about the behavior and attributes of the child is paramount. The aim of this paper is to present the results of the adaptation and validation of the Parent's Rating Scale of Child's Actual Behavior (PRS; Harter, 1985 ) for its use in Buenos Aires (Argentina). The sample of the study was compound by 155 children (age $M=9.53, S D=$ 1.40) of both genders from Buenos Aires elementary schools and one of their parents. Discriminant item capacity, reliability, construct, content, convergent, and discriminant validity were studied. The results showed that this is a valid and reliable instrument to assess parents' perceptions of the attributes and skills of their school age children in Buenos Aires. Key Words: Children Psychological Assessment; Scale; Behavior; Adaptation.

\section{Introducción}

La evaluación psicológica infantil tiene características que le son específicas. Una de ellas es que se trata de la evaluación de sujetos con un dominio relativo de conocimiento sobre ellos mismos. Los niños tienen un insuficiente logro de los mecanismos cognitivoafectivo-lógicos de autoconocimiento ya que se hallan en proceso de desarrollo y las características evolutivas de la actividad verbal, motora, y cognoscitiva pueden afectar la validez de la información que brindan de sí mismos (Forns, 1995). Es ampliamente

1 La correspondencia relacionada con este artículo debe enviarse a María Fernanda Molina. Dirección postal: Plaza 3038, PB “C” (C1430DHJ). Ciudad Autónoma de Buenos Aires, Argentina. Email: fer_molina@hotmail.com. 
reconocido que tienen dificultades para informar de manera ajustada sobre su comportamiento. Los profesionales dedicados a la evaluación infantil han señalado frecuentemente la tendencia de los niños a informar menores dificultades de las que poseen (Frick, Barry, \& Kamphaus, 2010). Esto lleva a preguntarse ¿En qué medida el niño sabe y puede informar acerca de lo que conoce de sí? Y ¿En qué medida ese conocimiento corresponde a la realidad? En la infancia la representación del sí mismo suele ser pobre, poco diferenciada, y expresada a través de elementos físicos (e.g., ser rubio) o de acción (e.g., jugar al fútbol; Forns, 1995).

Si bien, aún con esas limitaciones, la información que los niños pueden brindar sobre sí mismos debe que ser tenida en cuenta es preciso que sea contrastada con la visión de otros informantes. Es por ello que la evaluación infantil tiene que ser un proceso multi-método y multi-informantes, incluyendo además de los auto-informes, otras técnicas y otras fuentes de información (Forns, 1995; Kendall, \& Morris, 1991). Esto ha llevado a dar un valor considerable en la evaluación infantil a la valoración que pueden hacer los padres sobre las conductas y atributos del niño, debido a la importancia de la influencia parental en el comportamiento de sus hijos (Frick et al., 2010).

Para ello, existen múltiples instrumentos que permiten recabar información sobre el niño desde la perspectiva de los padres. Por ejemplo, las entrevistas estructuradas (e.g., KSADS-PL; Kaufman, Birmaher, Brent, Rao, \& Ryan, 1996), las escalas de amplio espectro (e.g., CBCL; Achenbach, 1991) y de síntomas específicos (e.g., SNAP IV; Swanson, 1992) son ampliamente utilizadas tanto en clínica como en investigación. Sin embargo, una evaluación completa de un niño requiere tener en cuenta no sólo sus déficits y dificultades sino también sus competencias (Cole, Gondoli, \& Peeke, 1998). En este sentido, el autoconcepto de los niños resulta de particular relevancia.

Este constructo es definido por Harter (1999) como los juicios valorativos sobre atributos dentro de dominios discretos como el cognitivo, el comportamental, social, entre otros, y tiene importantes consecuencias en el desarrollo infanto juvenil (Van Den Bergh, \& Marcoen, 1999). Por una parte, estas autopercepciones organizan las experiencias y guían los comportamientos (Swann, Chang-Schneider, \& McClarty, 2007). Por otra, una percepción del sí mismo saludable se asocia a logros de una relevancia incuestionable. Por ejemplo, un autoconcepto positivo se asocia a una mayor satisfacción vital (Huebner, 1991), resiliencia (Cardozo, \& Alderete, 2009), un desempeño académico favorable (Guay, Marsh, \& Boivin, 
2003), y una actitud positiva hacia la escuela y el estudio (Schmidt, Messoulam, \& Molina, 2008). Mientras que un autoconcepto negativo se asocia a una mayor predisposición al padecimiento de problemas internalizantes y externalizantes en general (Nishikawa, Sundbom, \& Hägglöf, 2010) y, en particular, a la depresión (Hoffman, Cole, Martin, Tram, \& Seroczynski, 2000; Jiménez, Pérez Murgui, \& Musitu Ochoa, 2007; Robins, Donnellan, Widaman, \& Conger, 2010), ideas suicidas (De la Torre, Cubillas Rodríguez, Román Pérez, \& Valdez, 2009; Rosselló, \& Berríos Hernández, 2004), ansiedad e inadaptación (Acevedo Ponce de León, \& Carrillo Árcega, 2010), y comportamiento riesgoso (uso de sustancia psicoactivas y conducta sexual riesgosa; Peterson, Buser, \& Westburg, 2010).

Por otra parte, el ajuste en la forma en que los niños se perciben a sí mismos también es importante. El desajuste en el autoconcepto, es decir tanto la subestimación como sobreestimación de competencias, se asocia a mayores niveles de agresividad (Brendgen, Vitaro, Turgeon, Poulin, \& Wanner, 2004; White, \& Kistner, 2011) y depresión (Kistner, David-Ferdon, Repper, \& Joiner, 2006). A su vez, un autoconcepto excesivamente elevado se asocia a menores logros terapéuticos (Mikami, Calhoum, \& Abikoff, 2010), dificultades para aprender de la experiencia y una menor motivación para cambiar (Owens, \& Hoza, 2003).

Por otra parte, desde una perspectiva clínica es crítico determinar si las autoevaluaciones negativas de los niños son realistas o no. Si el niño tiene deficiencias en áreas que deterioran su sentido de competencia y autoestima, es necesario intervenir directamente para desarrollar sus habilidades en esas áreas. En cambio, si sus autopercepciones son desproporcionadamente negativas, entonces en necesario intervenir directamente sobre ellas (Harter, 1999).

La Escala de Evaluación del Comportamiento del Niño (Harter, 1985) es un instrumento que ha sido diseñado para complementar la evaluación del autoconcepto de niños de 8 a 12 años. Más específicamente, a partir de su aplicación puede estudiarse el ajuste en la autopercepción de los niños así como obtener información para realizar una evaluación multiinformante de sus competencias. En su versión original, este instrumento fue diseñado para ser respondido por los docentes de los niños, pero Harter (1985) sugiere que puede ser adaptado para su uso con otros adultos, como los padres.

Esta escala ha sido utilizada en múltiples estudios. Por un lado, se la ha aplicado para evaluar el ajuste en el autoconcepto de los niños en distintas poblaciones. Por ejemplo, niños con Trastorno por Déficit de Atención con Hiperactividad (Hoza et al., 2004), depresión 
(Hoffman et al., 2000), o parálisis cerebral (Dunn, Shields, Taylor, \& Dodd, 2009). Asimismo, ha sido usado como parte de la evaluación multi-informante de las competencias del niño (Hutcherson \& Epkins, 2009; Shin, 2010; Tompkins \& Wyatt, 2008).

A pesar de que esta escala es considerablemente utilizada en la investigación en distintas temáticas vinculadas con la población infantil, hasta nuestro conocimiento existen escasos estudios que hayan indagado sus propiedades psicométricas. En primer lugar, son pocos los trabajos que hayan estudiado la estructura subyacente a esta escala. Según Harter $(1985,1999)$, en la infancia media son cinco los principales dominios del autoconcepto (competencias académicas, aceptación social por parte de los pares, competencias deportivas, apariencia física y comportamiento). La Escala de Evaluación del Comportamiento del Niño (PRS) se propone evaluar dichos dominios desde la percepción de los padres. Cole et al. (1998) por medio de análisis factorial confirmatorio, encontraron que la escala presenta una estructura factorial coherente con la estructura teórica en que se fundamenta su construcción, cargando todos los ítems en el factor hipotetizado. Por otra parte, en este trabajo se informa que la escala presenta una buena validez convergente y discriminante al comparar las respuestas de los padres con las de los docentes y pares de los niños (Cole et al., 1998). En cuanto a la confiabilidad, la escala ha mostrado tener una buena consistencia interna (Alfa entre .82 y .89) y una alta confiabilidad por test-retest con un intervalo de cuatro meses ( $r$ entre .60 y .80; Cole, Martin, Powers, \& Truglio, 1996; Seroczynski, Cole, \& Maxwell, 1997).

Hasta nuestro conocimiento, no existen trabajos que hayan explorado las propiedades psicométricas de este instrumento para su uso con niños de habla hispana. Es por esto que el presente trabajo se propone estudiar la validez y confiabilidad de los datos obtenidos con la PRS para su uso con niños de escuelas primarias de Buenos Aires.

\section{Método}

Participantes

La muestra fue obtenida de un estudio más amplio. Se trabajó con 155 niños (edad $M=9.53 ; D E=1.4$ ) de ambos sexos (varones: $61.9 \%$ ) de escuelas primarias de Buenos Aires (Argentina) y uno de sus padres (madres: $79.4 \%$ ). La mayoría de los padres estaban casados (69.3) y tenían un nivel de instrucción universitario (madres: 42.6\%; padres: 33.3\%). La 
mayoría de los niños pertenecía a un hogar biparental (75.2\%).

\section{Instrumentos}

Cuestionario sociodemográfico. Fue construido ad-hoc para caracterizar la muestra en función de sus características socio-demográficas (edad, nivel educativo y estado civil de los padres, composición familiar)

Escala de evaluación del comportamiento del niño. Versión para padres (PRS; Harter, 1985). Consta de 15 ítems que evalúan la percepción que tienen los padres de atributos y comportamientos del niño en cinco dominios: Competencias Académicas (percepción de los padres de la competencia del niño en la esfera del rendimiento académico); Aceptación Social (percepción de los padres del grado en que el niño es aceptado por sus pares o se siente popular); Competencias Deportivas (percepción de los padres de la capacidad del niño para los deportes o los juegos al aire libre); Apariencia Física (grado en que los padres perciben que el niño tiene un buen aspecto físico, un buen cuerpo, y un buen peso y altura); y Comportamiento (grado en que los padres perciben que el niño se comporta adecuadamente y evita meterse en problemas). Cada subescala se compone por tres ítems con cuatro opciones de respuesta. El formato de respuesta consiste en dos frases contrapuestas que muestran dos grupos de niños con comportamientos o competencias opuestas. Los sujetos deben elegir, en primer lugar, a qué grupo se parece el niño y, en segundo lugar, en qué grado se parece a ese grupo ("Realmente verdadero" o "En cierto modo verdadero"). Los ítems son del tipo: "Mi hijo/a encuentra difícil hacerse amigos $o$ Para mi hijo/a es bastante fácil hacerse amigos" (Aceptación Social) o "Mi hijo/a usualmente se comporta bien $o$ Mi hijo/a a menudo no se comporta bien" (Comportamiento)

Perfil de Autopercepciones para niños (SPPC; Harter, 1985; Adaptación Molina, Raimundi, López, Cataldi, \& Bugallo, 2011). Posee 36 ítems y evalúa el autoconcepto a través de los mismos dominios evaluados por el PRS. Cada subescala se compone de seis ítems con cuatro opciones de respuesta. El formato de respuesta consiste en dos frases contrapuestas que muestran dos grupos de niños con autopercepciones opuestas. Los niños deben elegir en primer lugar, a qué grupo se parecen y, en segundo lugar en qué grado se parecen a ese grupo ("Realmente como yo" o "Más o menos como yo"). Los ítems son del tipo: "Algunos chicos pueden aprender con facilidad lo que se les enseña en la escuela Pero Otros chicos tienen dificultad para aprender lo que se les enseña" (competencias académicas) 
o "Algunos chico sienten que son buenos para hacer actividad física Pero Otros chicos no son muy buenos para hacer actividad física" (Competencias Deportivas). En su versión original el SPPC ha mostrado tener una estructura factorial clara en la que cada uno de los cinco dominios específicos define su propio factor con pesajes sustanciales y mínimas cargas cruzadas. Por otra parte, las subescalas presentaron una adecuada consistencia interna (Alfa entre .71 a .86; Harter, 1985). La versión local de la escala mostró adecuados indicadores de validez y confiabilidad (Molina et al., 2011). En la muestra de este estudio la escala presenta buenos coeficientes de consistencia interna para las seis subescalas (competencia académica, $\alpha=.81$; aceptación social, $\alpha=.79$; competencia deportiva, $\alpha=.80$; apariencia física, $\alpha=.81$; comportamiento, $\alpha=.85)$.

\section{Procedimiento}

Se contactó a los padres de los niños por medio de una nota en el cuaderno de comunicaciones. Se les envió el cuestionario sociodemográfico y el PRS para ser enviadas nuevamente a la escuela en sobre cerrado. Uno de los padres completó el PRS. Los niños respondieron el SPPC en forma grupal. La consigna fue leída en voz alta y explicada por los evaluadores. Luego, los cuestionarios fueron respondidos en forma individual. La participación de los padres y los niños fue voluntaria y confidencial. Se obtuvo consentimiento escrito por parte de los padres.

\section{Análisis de datos}

Para analizar el funcionamiento de los ítems se realizó un análisis de discriminación, estudiando la correlación ítem-total corregida. Para estudiar la confiabilidad del instrumento se analizó la consistencia interna de las subescalas con el índice Alfa de Cronbach. Para probar la validez de constructo se realizó un análisis factorial confirmatorio. Para probar la adecuación del modelo se utilizaron índices de ajuste incremental y absoluto seleccionados en base a recomendaciones y convenciones (Browne, \& Cudeck, 1993; García Cueto, Gallo Álvaro, \& Miranda, 1998; Hair, Anderson, Tatham, \& Black, 1998; Jöreskog \& Sörbom, 1989; Schmidt, Barreyro, \& Maglio, 2009). Se seleccionaron (1) el índice RMR (Root Mean Residual), este índice minimiza el sesgo producido por el tamaño de muestra; (2) el índice RMSEA (Root Mean Square Error of Aproximation) mide la bondad de ajuste entre el modelo y los datos poblacionales. En ambos casos se considera que valores iguales o inferiores a .08 
indican un buen ajuste; (3) el índice GFI (Goodness of Fit Index) que representa el grado de ajuste conjunto del modelo sin tener en cuenta los grados de libertad; (4) El índice CFI (Comparative Fit Index) que mide el porcentaje de pérdida que se produce de cambiar el modelo predicho al modelo nulo en el ajuste; (5) El índice IFI (Incremental Fit Index) que además tiene en cuenta los grados de libertad del modelo; (6) El índice TLI (o índice de Tucker-Lewis) que es uno de los índices menos afectado por el tamaño de la muestra. En los último cuatro índices se considera que valores superiores a .90 indican un buen ajuste.

Con el objetivo de obtener evidencias adicionales a favor de la validez de constructo se realizó análisis de correlación para indagar las asociaciones intraescala ( $r$ de Pearson). Para obtener evidencias sobre la validez convergente y discriminante se estudió la correlación ( $r$ de Pearson) entre las respuestas de los padres al PRS describiendo a sus hijos, y las respuestas de éstos al SPPC describiéndose a sí mismos. Más específicamente, se estudiaron las correlaciones entre las respuestas de padres e hijos en las subescalas que miden un mismo constructo (validez convergente) y en las subescalas que miden constructos diferentes (validez discriminante; Hogan, 2004).

\section{Resultados}

Validez de contenido

Para aportar evidencia a favor de la validez de contenido del instrumento, se realizó la adaptación lingüística y conceptual. Se buscó que la escala fuera un buen reflejo de la versión local del SPPC (Molina et al., 2011) con el objetivo de poder comparar la percepción que el niño tiene de sí mismo con la que tienen sus padres. La escala fue valorada por cuatro jueces expertos en evaluación psicológica y en el trabajo con niños para conocer el grado de ajuste ítem-constructo, el grado de adecuación sintáctica y semántica, y el grado en que la escala reflejaba al SPPC. A partir de la evaluación de los jueces expertos se modificaron algunas expresiones para que fueran apropiados para nuestra cultura. De este modo, se logró adaptar lingüística y conceptualmente el instrumento, obteniéndose una primera versión local.

\section{Capacidad de discriminación de los ítems}

Se obtuvo una correlación ítem-total superior a .30 para todos los ítems, indicando que poseen una adecuada capacidad de discriminación (Tornimbeni, Pérez, \& Olaz, 2008). En la Tabla 1 se consignan los valores de discriminación de los ítems para cada subescala. 
Tabla 1.

Capacidad de discriminación de los ítems (correlaciones ítem-total)

\begin{tabular}{ccccccccccc}
\hline & \multicolumn{2}{l}{ Académica } & Social & & \multicolumn{2}{c}{ Deportiva } & \multicolumn{2}{c}{ Apariencia Física } & \multicolumn{2}{c}{ Comportamiento } \\
\hline & Ítem & Disc. & Ítem & Disc. & Ítem & Disc. & Ítem & Disc. & Ítem & Disc. \\
\hline 1 & .69 & 2 & .54 & 3 & .76 & 4 & .37 & 5 & .75 \\
& 6 & .67 & 7 & .65 & 8 & .71 & 9 & .54 & 10 & .76 \\
& 11 & .73 & 12 & .57 & 13 & .64 & 14 & .58 & 15 & .73 \\
\hline Alfa & .83 & & .75 & & .84 & & .66 & & .87 & \\
\hline
\end{tabular}

Las subescalas que componen el instrumento poseen índices de consistencia interna adecuados (alfa de Cronbach de .66 a .87; ver Tabla 1). En la mayoría de las subescalas, el coeficiente no aumenta al quitar algún ítem, salvo en la subescala de Apariencia Física, donde al quitar el ítem 4, el alfa aumenta a .69.

Validez de constructo

Análisis factorial confirmatorio. Se puso a prueba un modelo de medida de cinco factores latentes de primer orden que saturan tres ítems cada uno. Se utilizó el método de máxima verosimilitud. Se analizaron diferentes índices de ajuste (global e incremental). Todos ellos indicaron un excelente ajuste del modelo $(\mathrm{GFI}=.90 ; \mathrm{TLI}=.92 ; \mathrm{IFI}=.94 ; \mathrm{CFI}=$ .94; $\mathrm{SRMR}=.07 ; \mathrm{RMSEA}=.07)$. Las covarianzas entre los cinco factores son inferiores a .5, a excepción de la covarianza entre los factores correspondientes a competencias académicas y comportamiento, aportando a la validez discriminante entre los cinco factores latentes. En la Figura 1 se presenta el modelo con los pesos de regresión de las variables, que van desde .45 a .91 y las covarianzas entre los factores. 


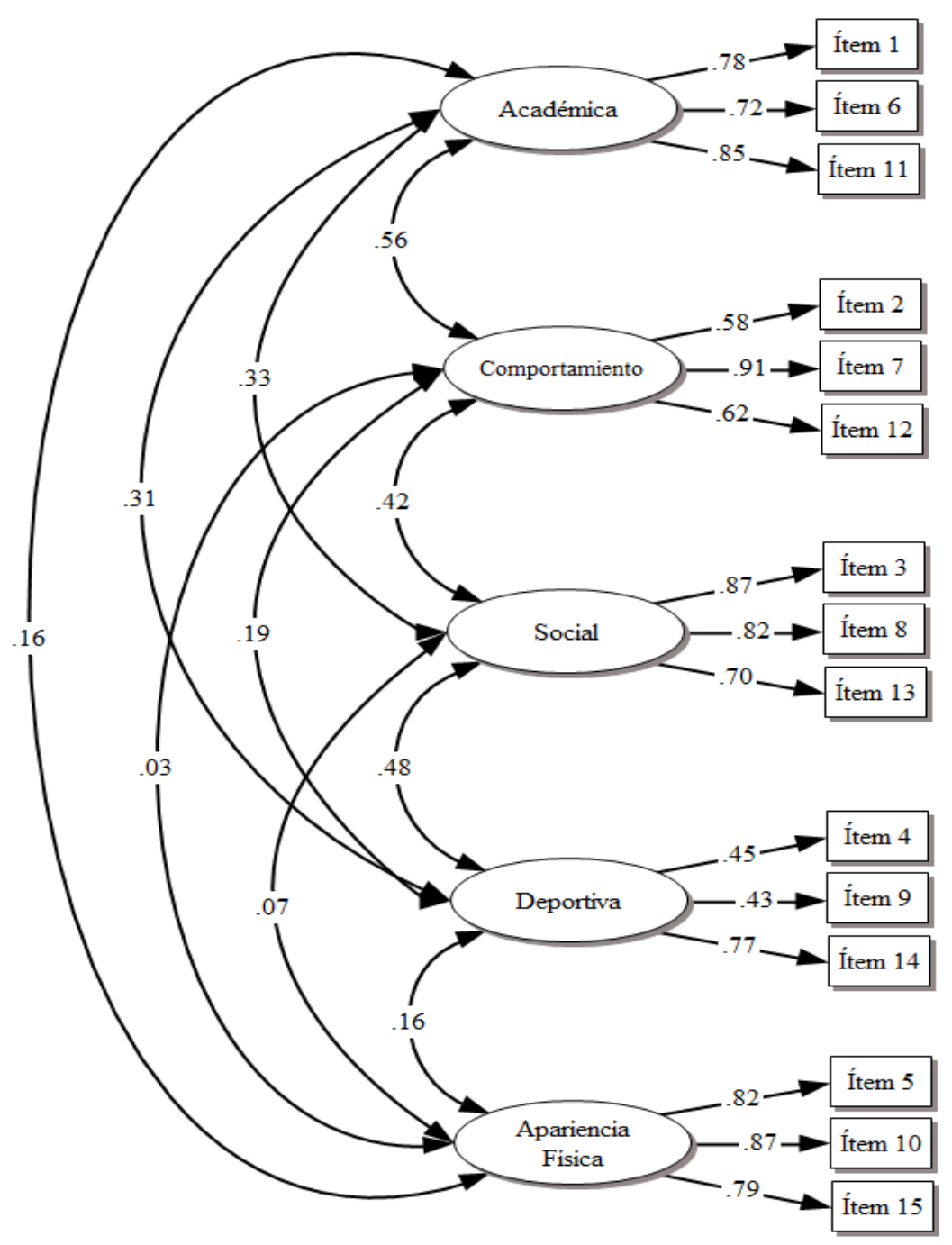

\section{Figura 1.}

Pesos de regresión y covarianzas del análisis factorial confirmatorio

Correlaciones intraescala. Se hallaron correlaciones intraescala positivas de bajas a moderadas (ver Tabla 2). Asimismo, se observan correlaciones significativas $(p<.05)$ y muy significativas $(p<.01)$ entre la mayoría de las subescalas. A partir de esto se puede concluir que se están evaluando variables relativamente independientes a pesar de que, en su mayoría, están algo relacionadas. 
Al examinar las correlaciones entre los dominios específicos se observan correlaciones muy significativas y moderadas entre Aceptación Social y Competencias Deportivas $(p<.01)$ y entre Competencias Académicas y Comportamiento $(p<.01$; ver Tabla 2).

\section{Tabla 2.}

Correlaciones intratest

\begin{tabular}{llllll}
\hline & 1 & 2 & 3 & 4 & 5 \\
\hline 1.Académica & - & $.21^{*}$ & $.28^{* *}$ & .14 & $.47^{* *}$ \\
2.Social & & - & $.41^{* *}$ & .07 & $.25^{* *}$ \\
3.Deportiva & & - & $.20^{*}$ & .14 \\
4.Apariencia Física & & & - & .02 \\
5. Comportamiento & & & & - \\
\hline
\end{tabular}

$* * p<.01 ; * p<.05$

Validez convergente y discriminante. En la Tabla 3 se presentan las correlaciones entre las respuestas de los padres al PRS y las de los niños al SPPC. Como evidencias a favor de la validez convergente, se hallaron correlaciones significativas positivas y moderadas entre las percepciones de los padres y las autopercepciones de los niños en el mismo dominio (e.g., competencias académicas). Como evidencias a favor de la validez discriminante, las correlaciones entre las percepciones de los padres y las autopercepciones de los niños en distintos dominios (e.g., entre competencias académicas y apariencia física) o bien: no fueron significativas, fueron bajas, o no tuvieron tanta fuerza como las correlaciones en un mismo dominio. 
Tabla 3.

Correlaciones entre percepciones de los padres y autopercepciones de los niños en los distintos dominios

\begin{tabular}{lccccc}
\hline & $\begin{array}{c}\text { SPPC- } \\
\text { Académica }\end{array}$ & $\begin{array}{c}\text { SPPC- } \\
\text { Social }\end{array}$ & $\begin{array}{c}\text { SPPC- } \\
\text { Deportiva }\end{array}$ & $\begin{array}{c}\text { SPPC-A. } \\
\text { Física }\end{array}$ & $\begin{array}{c}\text { SPPC- } \\
\text { Comportamiento }\end{array}$ \\
\hline PRS-Académica & $.46^{* *}$ & .12 & $.19^{*}$ & .10 & $.26^{* *}$ \\
PRS-Social & $.28^{* *}$ & $.43^{* *}$ & $.39 * *$ & .15 & $.20^{*}$ \\
PRS-Deportiva & .10 & $.32 * *$ & $.39 * *$ & $.19 *$ & -.05 \\
PRS- A. Física & .09 & -.05 & .08 & $.41^{* *}$ & .05 \\
PRS-Comportamiento & $.29 * *$ & $.19^{*}$ & .08 & -.01 & $.48^{* *}$ \\
\hline$* * p<.01 ; * p<.05$ & & & & &
\end{tabular}

\section{Discusión}

El presente trabajo tuvo como objetivo estudiar la validez y confiabilidad del PRS para su uso con niños de Buenos Aires. A partir de los resultados presentados se puede inferir que el PRS es un instrumento confiable y válido para evaluar la percepción que tienen los padres de niños de escuelas primarias de Buenos Aires de los atributos y competencias de sus hijos.

En primer lugar, se han encontrado evidencias a favor de la validez de contenido. A partir de la evaluación de jueces expertos se puede afirmar que la escala ha mostrado tener un adecuado ajuste ítem-constructo, ser apropiada para nuestra cultura y un reflejo adecuado del SPPC, desde el punto de vista conceptual.

Por otro lado, los ítems que componen la escala presentan una adecuada capacidad de discriminación.

En cuanto a la validez de constructo, se ha logrado confirmar la estructura de cinco factores de la versión original mediante el análisis factorial confirmatorio.

Asimismo, las correlaciones intraescala muestran que la escala mide constructos relativamente relacionados pero independientes entre sí. Sin embargo, resulta de interés señalar que se observa una asociación positiva y estadísticamente significativa entre las subescalas Competencias Académicas y Comportamiento, y Aceptación Social y Competencias Deportivas. Esta asociación ha sido encontrada en otros estudios, tanto respecto a las autopercepciones de los niños (Harter, 1985) como desde el punto de vista de sus padres, sus docentes (Cole et al., 1998) y sus pares (Cole, \& White, 1993). Según estos 
resultados, los niños que se perciben y son percibidos como competentes en el ámbito escolar también presentan una adecuada conducta desde su autopercepción y la de los demás.

Asimismo, el desempeño en el ámbito deportivo y de las habilidades físicas se relaciona con el grado en que el niño es aceptado por sus pares, tanto desde su propia visión como desde la valoración de los otros. Sin embargo, a diferencia de los resultados hallados en los estudios antes mencionados, en los que los dominios de Apariencia Física, Competencias Deportivas y Aceptación Social formaban un cluster en el sentido de que los tres dominios se asociaban entre sí, en este estudio la Apariencia Física no presentó una asociación significativa con Aceptación Social, y su correlación con Competencias Deportivas, si bien es positiva y significativa, es débil. Tal vez puede sostenerse a modo de hipótesis, que en la cultura anglosajona la importancia que tiene la apariencia física, y su relación con las competencias deportivas y la aceptación social, es mayor que la que existe en nuestro contexto.

En cuanto a la confiabilidad de la escala, los índices de consistencia interna son de adecuados a buenos mostrando que la escala es confiable para ser usada en nuestro medio. Sin embargo, cabe destacar que uno de los ítems de la subescala Apariencia Física disminuye la consistencia interna de la escala. Teniendo en cuenta que el valor de alfa, cuando se conserva el ítem, es adecuado, se decidió conservar dicho ítem debido a su importancia teórica. De todas formas, esta subescala requiere ser estudiada con mayor profundidad y los datos aportados por ella deben ser interpretados con cautela.

Por otra parte, se han encontrado evidencias a favor de la validez convergente del instrumento ya que las puntuaciones de los padres correlacionan positiva y significativamente con las autopercepciones de los niños en el dominio correspondiente. En un estudio de metaanálisis, ampliamente citado, realizado por Achenbach, McConaughy y Howell (1987) se encontró que la media de las correlaciones entre el informe de padres e hijos en distintas medidas del comportamiento y atributos de los niños es de .25 . Por otra parte, en un estudio transcultural en el que se indagó en 25 sociedades el acuerdo entre padres y adolescentes respecto del informe de los problemas psicopatológicos de los hijos, se encontró una correlación media omnicultural de .41 con un rango de .17 (Jamaica) a .58 (Dinamarca). Las correlaciones de los dos países latinoamericanos incluidos en el estudio citado son similares a las halladas en el presente trabajo (Perú .40 y Puerto Rico .43; Rescorla et al., 2012). El hecho de que las asociaciones en el informe dado por padres e hijos sean de moderadas a 
bajas no invalida la información por parte alguna de las fuentes, por el contrario, señala que los datos aportados por cada informante complementan los datos aportados por los demás (Achenbach et al., 1987; Van Den Bergh, \& Marcoen, 1999).

Asimismo, se han encontrado evidencias de validez discriminante de este instrumento ya que las correlaciones entre padres e hijos en diferentes dominios no son significativas, son bajas, o son más débiles que las correlaciones en el mismo dominio. Esto evidencia que no se observa una asociación entre las respuestas de padres e hijos en subescalas que miden constructos diferentes.

En cuanto a las implicancias de resultados aportados por este trabajo, el PRS es un instrumento ampliamente utilizado en investigación tanto para la evaluación multi-informante de competencias del niño como para la evaluación del ajuste en su autoconcepto. Este instrumento posee una gran utilidad tanto para su uso en investigación como en el trabajo con niños. Es por esto que es de suma relevancia contar con información sobre sus propiedades psicométricas para su uso en la evaluación de niños de nuestro contexto.

Por otra parte, el presente trabajo no está exento de limitaciones. En primer lugar, en este estudio se trabajó con una muestra no probabilística, por lo que la misma no es representativa y los resultados deben ser replicados con otras muestras. En segundo lugar, el tamaño de la muestra no permitió estudiar las propiedades psicométricas de la escala para las madres y padres por separado. Debido a la alta proporción de madres que respondieron la escala, existen limitaciones para generalizar los resultados para el uso de la escala con los padres. En futuras investigaciones, se deberá ampliar la muestra de padres para poder estudiar las propiedades de la escala para su uso específico con madres y padres.

Por otra parte, es importante destacar la importancia de validar este instrumento en su versión para docentes, debido a que el contexto escolar es uno de los más relevantes en esta etapa vital (Forns, 1995) y que los padres y docentes tienen la oportunidad de observar al niño en situaciones diferentes $\mathrm{y}$, por lo tanto, la información aportada por ambos es complementaria (Cole et al., 1998).

\section{Conclusiones}

A partir de lo expuesto, podemos concluir que la escala muestra adecuada capacidad de discriminación en sus ítems, confiabilidad aceptable, y evidencias de validez de constructo, de contenido, convergente y discriminante. De esta forma, la versión local del 
PRS muestra tener adecuadas propiedades psicométricas al ser utilizada en la evaluación de niños de escuelas primarias de Buenos Aires, a través de la percepción de sus padres. Asimismo, se logró un instrumento adecuado para ser utilizado como complemento del SPPC.

\section{Nota}

Esta investigación fue financiada por el Consejo Nacional de Investigaciones Científicas y Tecnológicas (CONICET; Res. No 3609/11 Director: Dra. Schmidt. Co-Director: Dra. Leibovich de Figueroa) y por la Secretaria de Ciencia y Técnica de la Universidad de Buenos Aires (UBACyT 20020100100052; Director: Dra. Leibovich de Figueroa. Co-Director: Dra. Schmidt).

\section{Agradecimientos}

Se agradece a los alumnos, padres, docentes y autoridades de la escuela que participó en este estudio su colaboración en este proyecto de investigación. Asimismo se agradece a las Lic. Mariel Giménez, Lic. Lucía Bugallo y Lic. Yanina Guzmán su colaboración en la recolección de datos para esta investigación.

\section{Referencias}

Acevedo Ponce de León, J. A., \& Carrillo Árcega, M. L. (2010). Adaptación, ansiedad y autoestima en niños de 9 a 12 años: una comparación entre escuela tradicional y Montessori, Psicología Iberoamericana, 18, 19-29.

Achenbach, T. M. (1991). Manual for Child Behavior Checklist/ 4-18 and 1991 Profile. Burlington, VT: University of Vermont, Dept. of Psychiatry.

Achenbach, T. M., McConaughy, S. H., \& Howell, C. T. (1987). Child/adolescent behavioral and emotional problems: implications of cross-informant correlations for situational specificity. Psychological bulletin, 101, 213-32.

Brendgen, M., Vitaro, F., Turgeon, L., Poulin, F., \& Wanner, B. (2004). Is there a dark side of positive illusions? Overestimation of social competence and subsequent adjustment in aggressive and nonaggressive children. Journal of Abnormal Child Psychology, 32, 305-320.

Browne, M. W., \& Cudeck, R. (1993). Alternative way of assessing model fit. En K. A. 
Bollen \& J. S. Long (Eds.), Testing Structural Equation Models (pp. 8136-8162). Newbury Park: Sage Publication.

Cardozo, G., \& Alderete, A. M. (2009). Adolescentes en riesgo psicosocial y resiliencia. Psicología desde el Caribe, 23, 148-182.

Cole, D. A., Martin, J. M., Powers, B., \& Truglio, R. (1996). Modeling causal relations between academic and social competence and depression: A multitrait-multimethod longitudinal study of children. Journal of Abnormal Psychology, 105, 258-270.

Cole, D. A., \& White, K. (1993). Structure of peer impressions of children's competence: Validation of the peer nomination of multiple competencies. Psychological Assessment, 5, 449-458.

Cole, D. A., Gondoli, D. M., \& Peeke, L. G. (1998). Structure and validity of parent and teacher perceptions of children's competence: A multitrait-multimethod-multigroup investigation. Psychological Assessment, 10, 241-249. doi:10.1037//10403590.10.3.241

De la Torre, I. M., Cubillas Rodríguez, M. J., Román Pérez, R., \& Valdez, E. A. (2009). Ideación suicida en población escolarizada infantil: Factores psicológicos asociados. Salud Mental, 32, 495-502.

Dunn, N., Shields, N., Taylor, N. F., \& Dodd, K. J. (2009). Comparing the self-concept of children with cerebral palsy to the perceptions of their parents. Disability and rehabilitation, 31, 387-393. doi:10.1080/13682820802052125

Forns, M. (1995) Evaluación Psicológica Infantil. Barcelona: Barcanova.

Frick, P. J., Barry, C. T., \& Kamphaus, R. W. (2010). Clinical Assessment of Child and Adolescent Personality and Behavior ( $3^{\circ}$ Ed.). New York: Springer. doi: 10.1007/9781-4419-0641-0

García Cueto, E., Gallo Álvaro, P., \& Miranda, R. (1998). Bondad de ajuste en el análisis factorial confirmatorio. Psicothema, 10, 717-723.

Guay, F., Marsh, H. W., \& Boivin, M. (2003). Academic self-concept and academic achievement: Developmental perspectives on their causal ordering. Journal of Educational Psychology, 95, 124-136.

Hair, F., Anderson, R. E., Tatham, R. L., \& Black, W. C. (1998). Multivariate data analysis with readings: New Jersey: Prentice Hall.

Harter, S. (1985). Manual for de Self-Perception Profile for Children. Denver: University of 
Denver.

Harter, S. (1999). The construction of the self: a Developmental perspective. New York: The Guilford Press.

Hoffman, K. B., Cole, D. A., Martin, J. M., Tram, J., \& Seroczynski A. D. (2000). Are the discrepancies between self- and others' appraisals of competence predictive or reflective of depressive symptoms in children and adolescents: A longitudinal study, Part II. Journal of Abnormal Psychology, 109, 651-662. doi: 10.1037/0021843X.109.4.651

Hogan, T. (2004) Pruebas psicológicas: una introducción práctica. México: Manual Moderno.

Hoza, B., Gerdes, A. C., Hinshaw, S. P., Arnold, L. E., Pelham, W. E., Molina, B. S. G., Abikoff, H. B., et al. (2004). Self-perceptions of competence in children with ADHD and comparison children. Journal of Consulting and Clinical Psychology, 72, 382391. doi:10.1037/0022-006X.72.3.382

Huebner, E. S. (1991). Correlates of life satisfaction in children. School Psychology Quarterly, 6, 103-111.

Hutcherson, S. T., \& Epkins, C. C. (2009). Differentiating parent- and peer-related interpersonal correlates of depressive symptoms and social anxiety in preadolescent girls. Journal of Social and Personal Relationships, 26, 875-897. doi: $10.1177 / 0265407509345654$

Jiménez, T. I., Pérez Murgui, S., \& Musitu Ochoa, G. (2007). Comunicación familiar y ánimo depresivo: el papel mediador de los recursos psicosociales del adolescente. Revista Mexicana de Psicología, 24, 259-271.

Jöreskog, K. G., \& Sörbom, D. (1989). LISREL7: User's reference guide. Mooresville: Scientific Software.

Kaufman, J., Birmaher, B., Brent, D., Rao U, \& Ryan N. (1996) Kiddie-Sads-present and lifetime version (K-SADS-PL). University of Pittsburgh School of MedicineDepartment of Psychiatry, Pittsburgh.

Kendall, P. C., \& Morris, R. J. (1991). Child therapy: Issues and recommendations. Journal of Consulting and Clinical Psychology, 59, 777-784.

Kistner, J.A., David, C.F., Repper, K., \& Joiner, T.E. (2006). Bias and accuracy of children's: Perceptions of peer acceptance: Prospective associations with depressive symptoms. 
Journal of Abnormal Child Psychology, 34, 349-361.

Molina, M. F., Raimundi, M. J., López, C., Cataldi, S., \& Bugallo, L. (2011). Adaptación del Perfil de Autopercepciones para Niños para su Uso en la Ciudad de Buenos Aires. Revista Iberoamericana de Diagnóstico y Evaluación Psicológica (RIDEP), 32, 5378.

Mikami, A. Y., Calhoun, C. D., \& Abikoff, H. B. (2010). Positive illusory bias and response to behavioral treatment among children with attention-deficit/hyperactivity disorder. Journal of Clinical Child and Adolescent Psychology, 39, 373-85. doi:10.1080/15374411003691735

Nishikawa, S., Sundbom, E., \& Hägglöf, B. (2010). Influence of perceived parental rearing on adolescent self-concept and internalizing and externalizing problems in Japan. Journal of Child and Family Studies, 19, 57-66. doi: 10.1007/s10826-009-9281-y

Owens, J. S., \& Hoza, B. (2003). The role of inattention and hyperactivity/impulsivity in the positive illusory bias. Journal of Consulting and Clinical Psychology, 71, 680-691. doi:10.1037/0022-006X.71.4.680

Peterson, C. H., Buser, T.J., \& Westburg, N. G. (2010). Effects of familial attachment, social support, involvement, and self-esteem on youth substance use and sexual risk taking. Family Journal: Counseling and Therapy for Couples and Families, 18, 369-376.

Rescorla, L., Ginzburg, S., Achenbach, T. M., Ivanova, M. Y., Almqvist, F., Begovac, I., Bilenberg, N., et al. (2012). Cross-informant agreement between parent-reported and adolescent self-reported problems in 25 societies. Journal of Clinical Child and Adolescent Psychology, 53, 37-41. doi:10.1080/15374416.2012.717870

Robins, R. W., Donnellan, M. B., Widaman, K. F., \& Conger, R. D. (2010). Evaluating the link between self-esteem and temperament in Mexican origin early adolescents. Journal of Adolescence, 33, 403-410.

Rosselló, J., \& Berríos Hernández, M. N. (2004). Ideación suicida, depresión, actitudes disfuncionales, eventos de vida estresantes y autoestima en una muestra de adolescentes puertorriqueños/as. Revista Interamericana de Psicología, 38, 295-302.

Seroczynski, D. A., Cole, D. A., \& Maxwell, S. E. (1997). Cumulative and compensatory effects of competence and incompetence on depressive symptoms in children. Journal of Abnormal Psychology, 106, 586-97.

Schmidt, V., Barreyro, J. P., \& Maglio, A. L. (2009). Escala de evaluación del 
funcionamiento familiar FACES III: ¿Modelo de dos o tres factores? Escritos de Psicología, 3, 30-36.

Schmidt, V., Messoulam, N., \& Molina, M. F. (2008). Autoconcepto académico en adolescentes de escuelas medias: Presentación de un instrumento para su evaluación. Revista Iberoamericana de Diagnóstico y Evaluación Psicológica, 25, 81-106.

Shin, H. (2010). Does depression moderate or mediate the relations between deficits in children competence and aggression? : A short-term longitudinal study of Korean. School Psychology International, 31, 331-352. doi: 10.1177/0143034310377139

Swann, W. B. Jr., Chang-Schneider, C., \& McClarty, K. L. (2007). Do people's self-views matter? American Psychologist, 62, 84-94.

Swanson, J. M. (1992). School-based assessments and interventions for ADD students. Irvine, CA: KC Publishing.

Tompkins, T. L., \& Wyatt, G. E. (2008). Child psychosocial adjustment and parenting in families affected by maternal HIV/AIDS. Journal of Child and Family Studies, 17, 823-838. doi:10.1007/s10826-008-9192-3

Tornimbeni, S., Pérez, E., \& Olaz, F. (2008). Intruducción a la Psicometría. Buenos Aires: Paidós.

Van Den Bergh, B. H., \& Marcoen, A. (1999). Harter's self-perception profile for children: Factor structure, reliability, and convergent validity in a Dutch-speaking Belgian sample of fourth, fifth, and sixth grades. Psychologica Belgica, 39, 29-47.

White, B. A., \& Kistner, J. A. (2011). Biased self-perceptions, peer rejection, and aggression in children. Journal of Abnormal Child Psychology, 39, 645-656. doi: 10.1007/s10802-011-9506-6 